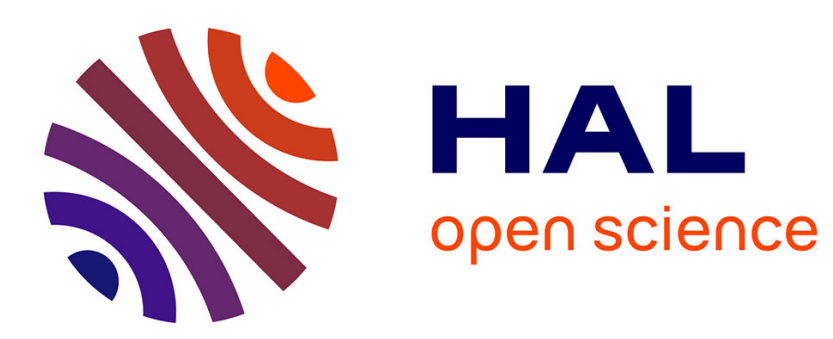

\title{
Acoustic emission during the solvent mediated cooling crystallization of citric acid
}

Xingjun Wang, Gilles Févotte, Ying Huang, Thomas Michelitsch

\section{To cite this version:}

Xingjun Wang, Gilles Févotte, Ying Huang, Thomas Michelitsch. Acoustic emission during the solvent mediated cooling crystallization of citric acid. Powder Technology, 2016, 301, pp.70 à 77. 10.1016/j.powtec.2016.05.057 . hal-01393432

\section{HAL Id: hal-01393432 \\ https://hal.science/hal-01393432}

Submitted on 10 Nov 2016

HAL is a multi-disciplinary open access archive for the deposit and dissemination of scientific research documents, whether they are published or not. The documents may come from teaching and research institutions in France or abroad, or from public or private research centers.
L'archive ouverte pluridisciplinaire HAL, est destinée au dépôt et à la diffusion de documents scientifiques de niveau recherche, publiés ou non, émanant des établissements d'enseignement et de recherche français ou étrangers, des laboratoires publics ou privés. 


\title{
Acoustic Emission During the Solvent Mediated Cooling Crystalliza- tion of Citric Acid
}

\author{
XingJun WANG ${ }^{* a, c}$; Gilles FEVOTTE ${ }^{\mathrm{a}, \mathrm{b}}$; Ying HUANG ${ }^{\mathrm{d}}$; Thomas M. MICHELITSCH \\ ${ }^{\text {a }}$ Ecole Nationale Supérieure des Mines, ENSMSE, centre SPIN, CNRS FRE 3312, LPMG, F-42023 St Etienne, France. \\ ${ }^{\mathrm{b}}$ Université Claude Bernard Lyon 1, Université Lyon 1, 43 boulevard du 11 Novembre 1918. 69622 Villeurbanne, \\ France. \\ ${ }^{\mathrm{c}}$ Qiannan Normal College for Nationalities, Department of Physics, LongShan Road, 5580oo Duyun, P.R.China \\ d Taizhou University, Collaborative Innovation Center, Shifu Road, 318000 Taizhou, P.R.China \\ e Sorbonne Universités, Université Pierre et Matie Curie, Paris 6, Institut Jean le Rond d'Alembert, CNRS UMR 7190, \\ Case 162, 4 Place Jussieu, 75252 Paris Cedex 05, France
}

\begin{abstract}
The potential of Acoustic Emission (AE) for controlling crystallization processes is investigated. The sensing technology is successfully applied to monitor the batch cooling crystallization of citric acid (CA) in water. The solventmediated phase transition between the anhydrous and the monohydrated forms of CA is clearly detected from the recorded acoustic measurements. A tremendous amount of acoustic data is recorded by the equipment, and the analysis of the data is focused on the evaluation of $\mathrm{AE}$ as a new sensor for monitoring the basic steps of the crystallization processes (i.e., nucleation, growth, phase transition, etc.) A time- and frequency-domain analysis is presented which shows the wealth of the technique. It is finally concluded that AE allows very early detection of nucleation events, provides a means of monitoring the development of the crystallization process and allows monitoring phase transition phenomena obtained through cooling. It is thus suggested that acoustic emission could be valuable in the development of new crystallization monitoring and control strategies: this is all the more interesting that the acoustic piezo-sensor is non-intrusive and does not require any sampling of the slurry, two features which are of tremendous importance in the field of cooling crystallization processes.
\end{abstract}

KEYWORDS: Solution Crystallization, Acoustic Emission, Monitoring and Control, Phase Transitions, Batch Processes, PAT.

\footnotetext{
* Phone number: +330658572316;

Email: xingjun.wang@qq.com; xingjun.wang@gmail.com
} 


\section{INTRODUCTION}

It is estimated that more than $80 \%$ of fine organic chemicals undergo at least one solid processing stage during their manufacture. In particular, as most active pharmaceutical ingredients (API) are processed and marketed in the solid form, solid particles elaboration and processing operations are essential in the fines chemical industry. In this particular industry as in other industries producing high value-added fine chemicals, crystallization is performed using batch processing which, owing to its unsteady-state dynamic features, requires continuous control during time. According to the products in question, crystallization can be essential as a solid-liquid separation, as a purification process, and also as a means of generating particles with specified end-use properties. Indeed, particle features such as the Crystal Size Distribution (CSD), crystal habit, chemical purity, crystallinity and polymorphic state are known to exert a significant impact on the end-use properties of the solid products (e.g., ease of downstream processing: filterability, behaviour on storage, flowability, etc.) This is the reason why improving the control over batch crystallization operations remains a major theoretical, technological and economic issue.

As far as advanced process monitoring is concerned, it is clear that until today no faultless measurement technique is available that would enable quantitative evaluation of quality variables characterizing the solid particles. In particular, this lack is clearly problematic for measuring in-line the CSD as all existing technologies, among which laser diffraction sensors, FBRM (Focused Beam Reflectance Measurement [1,2], image analysis) ... exhibit major limitations depending on the basic features of the slurry (e.g. the solid concentration, turbidity, etc.) and on properties of the particles (e.g. the particle shapes, the fines content, roughness, etc.) This is the reason why in situ sensors are actively being sought that would provide relevant information about the time evolutions of the quality of the solid product in suspension; and about the overall advancement of the process. Moreover, it should be outlined that developing sensing approaches in crystallization processes is all the more difficult that crystallization slurries are necessarily in non-equilibrium conditions: this makes it complex to withdraw solid/liquid samples for reliable external analyses.

Even though its process application in crystallization was not reported, acoustic emissions (AE) has shown potential as an efficient process analytical technology (PAT) for monitoring many industrial organic processes, in particular solids elaboration processes. Indeed, changes in physical properties related to both the progress of the process and the time variations of the product quality are likely to generate acoustic emission. This is why, from an industrial point of view, $\mathrm{AE}$ offers valuable features which are all the more promising that the technique is non-intrusive, non-destructive, and rather easy to implement.

In the past $\mathrm{AE}$ has widely been used in many field of process engineering. As far as active acoustic measurement techniques are concerned, a general review paper was published by [3]. Meanwhile, rather few applications of passive AE measurement dedicated to the monitoring and control of industrial processes were reported [4]. To speak only of pharmaceutical processes, and without being exhaustive, the correlation between audible acoustic emission and particle sizes was investigated by [5]. Several case-studies and review papers present AE applications in many fields of pharmaceutical process engineering: grinding [6], end-point detection in drying [7-9,5], blending and mixing [10,11], drying [12,13], fluidized beds [14], granulation [12,15], tabletting [16-18].

The technique of $\mathrm{AE}$ uses a transducer acoustically coupled to a process in which materials are undergoing dynamic changes. The sensor detects the elastic energy of acoustic waves propagating from the physical source of AE. The latter arises from mechanical or physical phenomena (fracture, plastic deformation, phase transition, delamination, etc.). Because one single crystal, considered as a physical source of $\mathrm{AE}$, can generate $\mathrm{AE}$, the acoustic signal emitted by developing slurries consists of a mixture of many waves, stretching over several orders of magnitude. This is why the recorded acoustic emissions frequency spectra are generally complex. One can therefore reasonably expect that, depending on the phenomena of interest, relevant information can be extracted, e.g. through frequency and/or multivariate statistical analyses.

As already outlined, a major advantage of the $\mathrm{AE}$ technique is that the sensors do not need to be placed inside solids processing equipments (e.g., reactors, dryers, granulators, grinders, crystallizers, etc.) The technology can therefore be used in hard process conditions, for example in high temperature and/or high pressure conditions, corrosive media and outside environments where the growth of pathogenic organisms takes place. Another advantage of AE lies in the possibility of collecting a wide range of information in real-time.

As far as crystallization is concerned, thanks to the large amount of collected data, one can reasonably expect AE to help designing new approaches for gaining a new point of view on basic crystallization phenomena (i.e. nucleation, growth, agglomeration ...), to increase process understanding and provide a basis for innovative online monitoring and control applications. Wade [19] proposed that during crystallization in solution local physicochemical changes are induced in the suspension, yielding release of energy. AE waves would be thus generated and propagate in the liquid medium. It is clear, for example, that when crystal particles are generated, the elastic properties of dispersed phase change. Inter-particles and/or particles-wall frictions can also occur and generate size-dependent acoustic emission. Other particle properties such as shape, hardness or brittleness can also affect the elasticity of the dispersed phase and its kinetic energy. 
In such a context this work aims at evaluating the potential for using acoustic emission to monitor batch cooling solution crystallization processes. In particular the ability of the AE technology for detecting specific event such as nucleation phenomena or phase transition is assessed. With this aim in view, the cooling crystallization of citric acid (CA) in water was selected as a model-system. Contrary to a previously reported study performed with ammonium oxalate in water [20], the present system offers the possibility of working with highly concentrated suspensions and to study solvent mediated phase transition phenomena occurring around ambient temperature.

\section{INTRODUCTION TO ACOUSTIC EMISSION}

The AE equipment consists of a piezoelectric sensor fixed on the wall of the crystallizer [21,22]. The sensor is connected to a fast data acquisition system dedicated to the processing of the many recorded pseudo periodic acoustic signals [23]. The latter are generated by the numerous crystals in suspension which are assumed to behave like tiny physical sources of emission. The acoustic signals propagate via the solution, the crystallizer wall and the heating/cooling external fluid, to the sensor where they are converted into electronic information. For further data processing, the electronic signal is converted into numerical information.

Acoustic emission waves appear as a sequence of a considerable number of "bursts" which are schematically illustrated in Fig.1. Some important features of the waves are explained in Fig.1. In order to differentiate significant hits from unavoidable noise, a reference level of energy (about 600 atto-Joules) and a threshold power ratio value (40 $\mathrm{dB}$ ) have to be determined first from "blank" measurements performed to evaluate the level of insignificant signals emitted e.g. by the electromagnetic environment, the stirring system and the flow of coolant fluid in the jacket of the crystallizer.



Figure 1. Main characteristic parameters of a typical AE burst.

Rather than recording the many raw acoustic measurements (several Go/h), global AE parameters can be computed to avoid recording too large a number of data. For example, a given acoustic wave can be characterized after integrating the output signal exceeding a given threshold or after computing its average and/or the number of counts $n$. (see Fig.1). Such global parameters characterizing the waves are called descriptors. The integrated Absolute Energy (IAE) is a measure of the true energy derived from the integral of the squared voltage signal emitted by the sensor, divided by the reference resistance (1o $\mathrm{k} \Omega$ ) over the duration of the AE signal. Another basic parameter used to characterize the level of acoustic signals in the time domain is the root mean square value (RMS) defined as the integral over time of the square of the pressure magnitude, $\mathrm{p}(\mathrm{t})$, of the acoustic wave. Working with descriptors is made easier than working with raw pseudoperiodic data, and less CPU time is spent because the descriptors can be processed without requiring prohibitory amounts of data. Some definitions of important descriptors are introduced in the following.

As displayed in Figure $1, \mathrm{p}(\mathrm{t})$ is actually evaluated from the electrical voltage $\mathrm{u}(\mathrm{t})$ emitted by the acoustic transducer and amplified by the sensing device, such that: $u(t) \propto p(t)$.

The amplitude AdB is defined as the maximal voltage of the AE signal divided by the reference voltage of the sensor which, in our case, is equal to $1 \mu \mathrm{V}$. It is thus defined as follows:

$$
A_{d B}=20 \log \left(u_{\max } / u_{r e f}\right)
$$

The average frequency $\bar{f}$ is defined as the average ratio between the number of counts $\mathrm{n}$ and the duration of the burst: 


$$
\bar{f}=n / d
$$

The peak frequency $\hat{f}$, expressed in $\mathrm{kHz}$, is defined as the point in the power spectrum at which the peak magnitude is observed, and the frequency centroid $\tilde{f}$, also in $\mathrm{kHz}$, is the barycentre computed from the sum of the frequencies balanced by the magnitude of the wave, it is equivalent to a first moment of inertia:

$$
\tilde{f}=\sum_{i} f_{i} E_{i} / \sum_{j} E_{j}
$$

The absolute energy, in atto-Joule $(1 \mathrm{aJ}=10-18 \mathrm{~J})$ is defined from the integration of the output voltage $\mathrm{u}(\mathrm{t})$ of the transducer as follows, $\alpha$ being inversely proportional to the electrical resistance of the measuring circuit :

$$
E_{a b s}=\alpha \int u(t)^{2} d t
$$

The cumulative absolute energy, referred below to as $\int E_{a b s}$ is obtained through the integration of $E_{a b s}$ over the time period $\tau=0$ to $t$ :

$$
\int E_{a b s}(t)=\int_{0}^{n(t)} E_{a b s}(\tau) d \tau
$$

\section{MATERIAL AND METHODS}

\subsection{Experimental setup}

A schematic of the crystallization setup used during the present study is displayed in Figure 2. Batch cooling seeded experiments were performed. The slope of the decreasing setpoint trajectory was set constant but, as explained in more details below, this did not help the cooling process to deviate from its setpoint trajectory in the presence of significant thermic effects. The crystallization was initiated thanks to the introduction of small amounts of anhydrous or monohydrate citric acid seed particles (less than $1 \mathrm{wt}^{\circ} \%$ ) at a temperature depending on the desired initial polymorphic state (i.e., anhydrous or monohydrate) and on the initial solute concentration. Such an amount of seeds is paltry: it was intentionally used to master the starting of the crystallization process and the phase of the crystallizing solid without avoiding secondary nucleation phenomena. The crystalline state of the seeds was checked using Raman spectroscopy.

The experiments were performed in a $2 \mathrm{~L}$ glass vessel equipped with a jacket. The jacket was baffled and the pump of the cooling bath forced the circulation. Stainless-steel baffles and a high efficiency propeller (Mixel TT TM) were used to maintain a good homogeneity of the suspension. The stirring rate was kept constant at $300 \mathrm{rpm}$. The bench-scale plant was instrumented and computer-controlled to allow tracking linear set-point temperature trajectories. Cooling was performed by means of heat transfer through the jacket wall. Both the temperatures of the crystallization medium and of the liquid circulating in the jacket were measured. In the sequel the temperature of the crystallizing slurry is referred to as $T(t)$. More details about the overall experimental design can be found in [24].

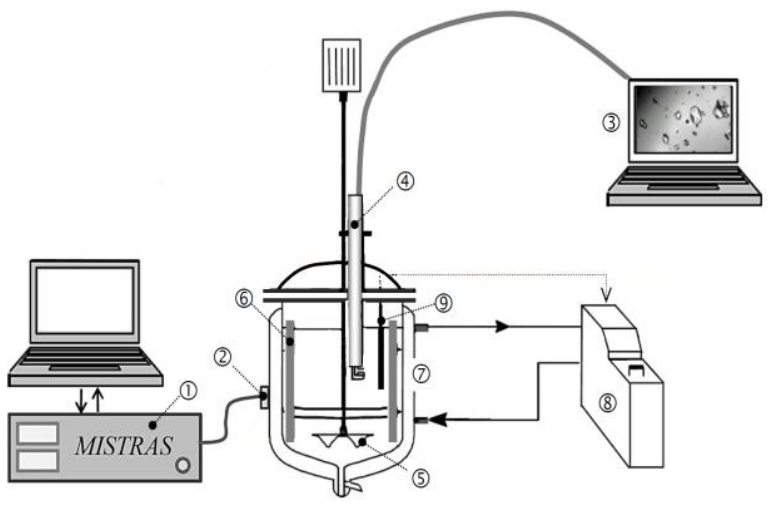

Figure 2: Experimental set up: temperature controlled and well-mixed batch crystallizer equipped with in situ image acquisition probe (4) and acoustic emission sensor (2). (1) Acoustic data acquisition system, (3) Computer dedicated to in situ image acquisition and processing, (5) Profiled mixing propeller, (6) Baffles, (7) Cooling jacket, (8) Heating/cooling bath, (9) Temperature probe. 
3.2 The model solute/solvent system

Citric acid can crystallize in water as anhydrous or monohydrate between $0^{\circ} \mathrm{C}$ and $60^{\circ} \mathrm{C}$. The two solid forms are referred to as $\mathrm{CA}_{\mathrm{a}}$ and $\mathrm{CA}_{\mathrm{m}}$ in the following. $\mathrm{CA}_{\mathrm{m}}$ was commercially available from Acros Organics with a purity level of $99+\%$. The powder was used without further purification. Crystallization experiments were performed in distilled water. $\mathrm{CA}_{\mathrm{a}}$ was initially dissolved in water with a concentration of $2.9 \mathrm{~kg}$ anhydrous $/ \mathrm{kg}$ water. Figure 3 displays the solubility curves of $\mathrm{CA}_{\mathrm{m}}$ and $\mathrm{CA}_{\mathrm{a}}$. The initial concentration corresponds to a saturation temperature of about $65^{\circ} \mathrm{C}$. It is worth noting that $\mathrm{CA}_{\mathrm{a}}$ is more stable above the transition point located closed to $35^{\circ} \mathrm{C}$, while $\mathrm{CA}_{\mathrm{m}}$ is more stable below.



Figure 3. Solubility diagram of citric acid in water. Data after [25] (1) Initial solution; (2) Seeding with $\mathrm{CA}_{\mathrm{a}}$; (3) Transition point $\left(35^{\circ} \mathrm{C}\right) ;(4)$ Solvent-mediated phase transition (The solubility curve of $\mathrm{CA}_{\mathrm{a}}$ is crossed around $\left.32^{\circ} \mathrm{C}\right)$; $(5)$ End of the crystallization of $\mathrm{CA}_{\mathrm{m}}$.

\subsection{Acoustic emission}

As shown in Figure 2, AE signals were collected using a piezoelectric sensor fixed on the wall of the crystallizer. Coupling grease was used to improve the transmission of acoustic waves from the crystallizer to the sensor. The acoustic signal was then conditioned, amplified, filtered and processed by a specific fast Data Acquisition System (DAS from EuroPhysical Acoustics S.A) to which the probe is connected. Table 1 summarizes the mains features of the AE equipment.

As explained above many AE descriptors can be acquired by the system, and recorded for further data processing. To differentiate significant hits from noise, a reference level of energy (i.e., $600 \mathrm{aJ}=610^{-16} \mathrm{~J}$ ) together with an appropriate threshold power ratio value $(40 \mathrm{~dB})$ were selected and set.

Four typical acoustic waves are presented in Figure 4. It should be noted that the sequence of these waves is almost continuous. In other words, it is rather difficult to isolate separate bursts (i.e. like the one displayed in Figure 1) from the alternating AE signal. This is the reason why the tuning parameters allowing distinguishing between two successive bursts should be carefully tuned, prior to any relevant acquisition and computation of the descriptors.
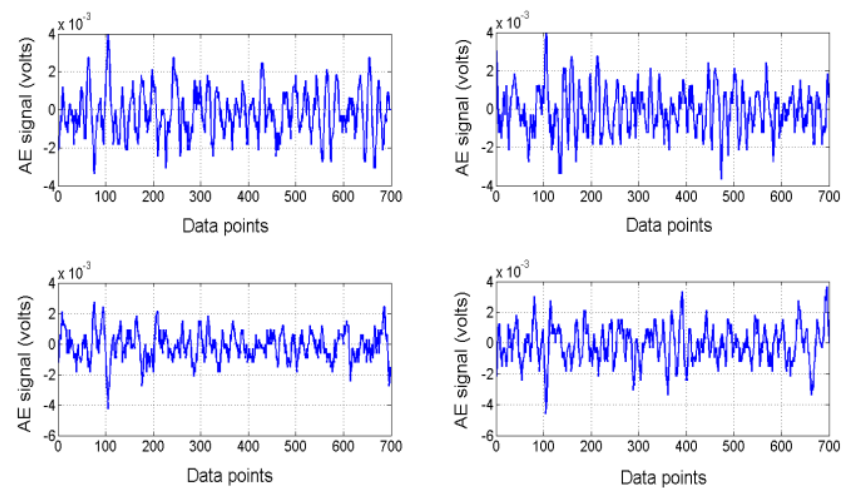

Figure 4. Typical acoustic bursts recorded during the crystallization of citric acid.

Table 1: Characteristics and set-up parameters for the AE acquisition system. 


\begin{tabular}{|c|c|}
\hline Instrumentation & Characteristics and set up \\
\hline DAS unit & AEDSP 32/16-2 \\
\hline Threshold $(\mathrm{dB})$ & 40 \\
\hline Sensor type: & $\begin{array}{c}\text { Physical Acoustics Corpo- } \\
\text { ration, WD FS62 }\end{array}$ \\
\hline Peak Frequency $(\mathrm{KHz})$ & 541.99 \\
\hline Preamplifier filter $(\mathrm{KHz})$ & $20-1200$ \\
\hline Preamplifiers $(\mathrm{dB})$ & 40 \\
\hline PDT-HDT-HLT $(\mu s)$ & $100-300-100$ \\
\hline
\end{tabular}

\section{Experimental results and discussion}

Given the many acoustic parameters described above it can be expected that relationships between significant acoustic variables and basic crystallization phenomena be established using appropriate data processing techniques. The point is to assess how these parameters can be analysed as a function of the development of the basic mechanisms occurring during the cooling process. The latter phenomena were simulated using Population Balance Equation (PBE) model and in situ Raman measurements of the solute concentration $C(t)$. A previous monitoring and modelling study of the system under investigation was reported by [26]. Such simulation, even though it is not explicitly used here, allows understanding the main features of the solute concentration trajectory with respect to phase transition events.

To obtain information from the many acoustic parameters, several methods such as time-domain analysis, frequency domain analysis and state-space analysis can be used [13]. Time-domain analysis is generally insightful to generate an overall view of the data over time, but it is rather uneasy to observe small changes from the raw data alone. With fast Fourier transformation (FFT), frequency domain analysis has the ability to inspect the frequency distribution over a domain of half of the sampling frequency of the measured signals. It allows measuring the frequency domain signals and scrutinizing possible changes in the frequency pattern over time (the transient power spectrum). Both time-domain analysis and frequency-domain analysis were used in this work.

Batch cooling crystallization of citric acid with solvent-mediated phase transition $\mathrm{CA}_{\mathrm{a}} \rightarrow \mathrm{CA}_{\mathrm{m}}$

Cooling crystallization experiments were carried out as described above. The ability of AE to detect secondary nucleation phenomena induced through seeding was first evaluated. Cooling solution crystallization experiments were performed with a cooling rate $\mathrm{R}=-\mathrm{dT} / \mathrm{dt}=-15^{\circ} \mathrm{C} / \mathrm{h}$, from $\mathrm{T}=59^{\circ} \mathrm{C}$ to $15^{\circ} \mathrm{C}$. The measured temperature profile is displayed in Figure 5

Due to the poor heat transfer capacity of the two glass walls of the jacket, the linear target temperature trajectory cannot be met when significant exothermal processes occur during the batch cooling process. Due to the heat of crystallization, the linearity of the temperature profile is lost when massive crystallization takes place. As already outlined and as described e.g. in Caillet et al. [27], the cooling batch crystallization of citric acid can give rise to the anhydrous to monohydrate solvent mediated phase transition in the temperature conditions of the actual experiments. From this point of view Figure 5 clearly exhibits two temperature bumps that can be related to the successive crystallization of anhydrous and monohydrate CA during the cooling process.

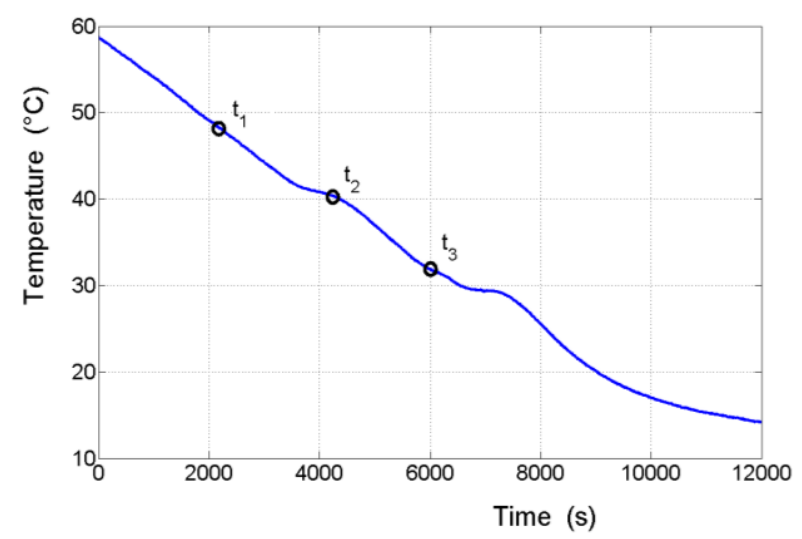


Figure 5. Temperature vs. time trajectory of a crystallization exhibiting $\mathrm{CA}_{\mathrm{a}}$ to $\mathrm{CA}_{\mathrm{m}}$ phase transition. The times delineating four specific acoustic emission zones are presented in Figure $7: t_{1} \cong 2100 \mathrm{~s} ; t_{2} \cong 4200 \mathrm{~s} ; \mathrm{t}_{3} \cong 6000 \mathrm{~s}$.

In order to get an idea of the development of the crystallization with anhydrous to monohydrate phase transition, a qualitative trajectory of the concentration of citric acid was plotted in Figure 3. Two temperature "bumps" can be observed in Figure 5 which are obviously related to the two successive crystallization processes involved during the batch cooling operation. The temperature evolution between $t_{1}$ and $t_{2}$ (i.e., from 49 to $40^{\circ} \mathrm{C}$ ) should be viewed in parallel with the acoustic events displayed in the following Figure 6 where the time period $\left[t_{1} t_{2}\right]$ is referred to as $\beta$. During the preceding time period $\alpha$, almost no acoustic activity has been recorded. During the time period $\delta \cong\left[t_{2} t_{3}\right]$, Figure 6 shows that the overall acoustic activity is reduced (i.e., in terms of number of counts) and that the frequencies of the recorded waves reduces to the bands $f_{6}$ to $f_{9}$ : the disappearance of frequency bands $f_{1}$ to $f_{5}$ is surprisingly sudden and clear.

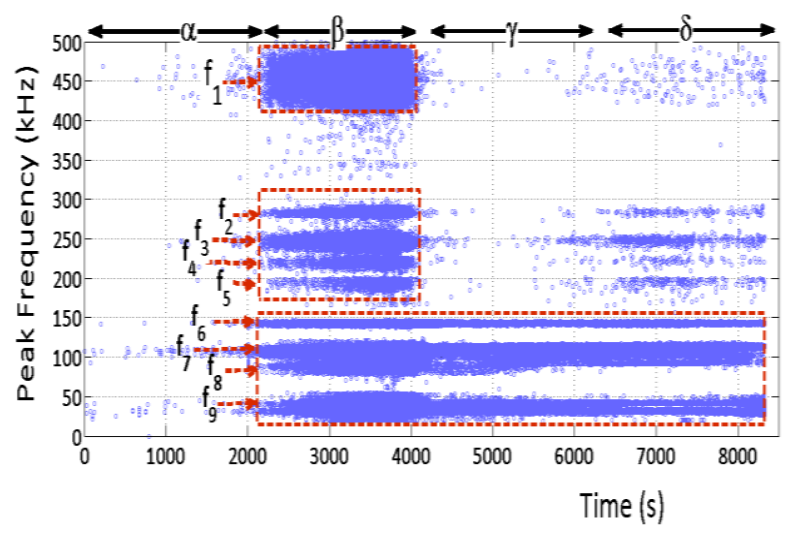

Figure 6. Time variations of the individual peak frequencies during the crystallization of citric acid. Nine main frequency bands are outlined by dashed boxes.

A scenario for the crystallization process with solvent-mediated phase transition is now proposed to support a possible explanation of the acoustic activity. Figure 7 was drawn to make the comparison between the various experimental results easier. A first difficulty raised by these results is the unexpected shift between "period $\beta$ " and the experimental temperature profile. Fig. 6 shows that the onset of the acoustic activity takes place around $t_{1}=2000 \mathrm{~s}$ (even a little bit earlier) while the temperature increase associated with the development of anhydrous crystallization starts around $t=3300 \mathrm{~s}$. Visual observation of the crystallizing slurry shows that many crystals are in fact generated during period $\beta$ : it makes no doubt (see Figure 3) that the highest level of supersaturation and, consequently, the highest nucleation rates are occurring during the same period. Now, during period $\gamma$, as already outlined, frequency bands $f_{1}$ to $f_{5}$ are no more active (i.e., crystal growth now dominates the overall crystallization process), while the remaining supersaturation — with respect to the anhydrous from of CA - is still positive. It seems therefore reasonable to assume that bands $f_{1}$ to $f_{5}$ could be indicative of the nucleation process. This latter assumption is consistent with the fact that bands $f_{6}$ to $f_{9}$ can be observed during the whole batch duration. Crystal growth is indeed the only permanent basic crystallization process from time $t_{1}$ to the end of cooling. 


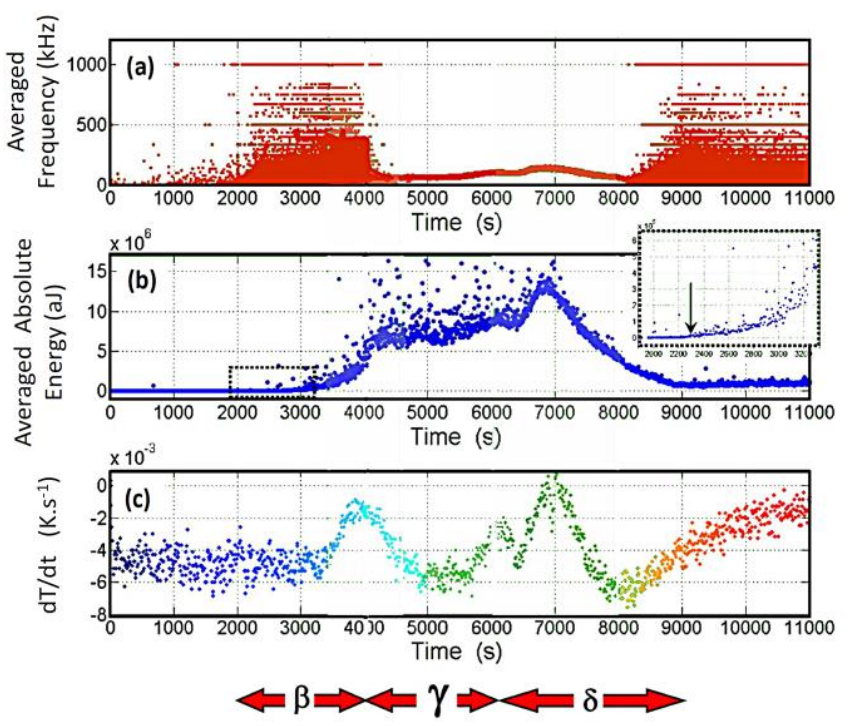

Figure 7. Comparison between the thermal effect of the development of the crystallization process and both frequency and absolute energy of the acoustic waves. Time periods $\alpha$ to $\delta$ refer to Fig. 7 and times $t_{1}$ to $t_{7}$ refer to Figs. $5 \& 6$.

Figure 7 compares the development of thermal effect of crystallization with the time variations of both the average frequency and the average absolute energy. Displaying the whole set of absolute energy data (i.e., clouds similar to the dots in Figure 6) would lack of clarity and, anyway, could not give a clear idea of the overall trends of the distribution of $\mathrm{AE}$ energy. This is why the absolute energy of the acoustic events was averaged as follows, where $N_{k}$ is the total number of hits recorded during in sample $n^{\circ} \mathrm{k}$, the sampling period being 3 seconds:

$$
\bar{E}_{a b s, k}=\frac{\sum_{k=1}^{N_{i, j}} E_{a b s}(k)}{N_{k}}
$$

$\bar{E}_{a b s, k}$ is plotted in Figure $7 \mathrm{~b}$, the plot shows that the acoustic activity becomes significant at the approximate time of 2250 s. (see the insert) The same processing was made with the frequency data. The results are plotted in Figure 7a.

Now, one could object that stirring, through inter-particles and particle/propeller shocks, could be the source of permanent emission. This possibility of acoustic emission dominated by stirring was evaluated. Actually, stopping the stirring device did not help acoustic emission to go on, which is not very surprising : given the very high solute concentration involved in this case, it is clear that, due to the high viscosity of the solution, the many shocks occurring in the suspension are so strongly damped that they can hardly generate waves. Consequently, even though this is far from being proved, it is suggested again that the acoustic activity in the frequency domain could be due to CA nucleation. This assumption would also explain why the nucleation of $\mathrm{CA}_{\mathrm{m}}$ (i.e., after time 6ooos) gives rise again to a sequence of acoustic activity in the same frequency domain $f_{1}$ to $f_{5}$. According to the previous assumption, the disappearance of "high frequency" activity during period $\gamma$ could be attributed to the fact that the nucleation events are here negligible (i.e. especially during the dissolution of $\left(\mathrm{A}_{\mathrm{a}}\right)$. At the end of period $\gamma$, the solute concentration $C(t)$ would thus cross the anhydrous solubility curve and go on decreasing until the final temperature where the solubility of the monohydrated form is finally met, as represented in Figure 3.

Figure 6 displayed the pseudo-frequencies of individual acoustic bursts detected by the piezo-sensor. It is now interesting to determine to which extent the successive crystallizations of anhydrous and monhydrate CA forms could give rise to specific variations of energy features of the acoustic data. From Figure 6, it appeared that the time variations of the AE frequency can roughly be divided into four parts. It should however be noted that, in terms of energy, the observation of dense clouds of $(\mathrm{t}, f)$ points does not mean that the emission is energetically sustained. In other words, the acoustic activity should also be considered with respect to its energy. Such analysis is now proposed.

During period $\beta$, it was outlined that the thermal signature of the first crystallization (i.e., crystallization of CAa) starting at time $t_{1} \cong 3500 \mathrm{~s}$ was significantly shifted with respect to the detection of acoustic bursts. Meanwhile, from a physical viewpoint, the first nucleation events cannot have any thermal effect on the reactor content because, due its size, the mass of nuclei is negligible. One can thus conclude that the nucleation of $\mathrm{CA}_{\mathrm{a}}$ occurs much before time $\mathrm{t}_{1}$ : this is consistent with the hypotheses made above.

During the first "exothermic period", the maximum increase of temperature occurs at $t_{3} \approx 4000$ s. The temperature trajectory corresponds to the energy released by the growth of anhydrous citric acid which cannot fully be compensated by 
heat transfer through the jacket wall. As already outlined, the AE sensor detects acoustic events very early: when AE occurs, no crystals can visually be observed in suspension and no heat release due the generation of solid is found to take place. Acoustic events exhibiting frequencies higher than $150 \mathrm{KHz}\left(\mathrm{f}_{1}\right.$ in Figure 6) appear to be specific of this period and, consequently, of the generation of $\mathrm{CA}_{\mathrm{a}}$.

As already outlined, a comparison between the temperature variations and the simultaneous acoustic emission highlights significant time mismatches. To get an additional point of view on the crystallization process, a more refined time and frequency domain analysis was performed. The frequency space was shared into the three main domains of interest underlined in Figure 6: [0-150 KHz], [150-300 KHz], and [300-500 KHz]. In order to reduce the amount of acoustic data and to compute a smooth $\left(\mathrm{t}, \bar{f}, a \bar{E}_{i, j}\right)$ surface plot, every time domain was divided into $120 \mathrm{~s}$ samples. The frequency range was divided into samples of width $4 \mathrm{KHz}$. The size of the time-frequency grid is therefore [50x70], the frequency and time domains being divided into 50 intervals $(300-500 \mathrm{KHz})$ and 70 intervals (o-8400 seconds), respectively. Thus, the time-frequency domain was cut in 3500 cells.

Every rectangular time-frequency cell (referred below to as $[i, j]$ ) is filled by the corresponding absolute energy value $a \bar{E}_{i, j}$ computed as follows:

$$
a \bar{E}_{i, j}=\frac{\sum_{k=1}^{N_{i, j}} E_{k}}{\Delta f . \Delta t}
$$

where $E_{k}$ is the value of the absolute energy of hit $\mathrm{n}^{\circ} \mathrm{k}$ located inside cell [i, j] and $N_{i, j}$ is the total number of hits inside the same cell. $\Delta f$ and $\Delta t$ are the frequency and time intervals defining the size of the $[\mathrm{i}, \mathrm{j}]$ grid.

Examining the surface plot $a \bar{E}_{i, j}=f(t, \bar{f})$ provides a clearer view on phenomena occurring during development of the crystallization process. Figure 8 shows the $3 \mathrm{D}$-plot of the average absolute acoustic energy recorded in the frequency domain [300-500] kHz. The plot corresponds to the time evolution of the frequency band $f_{1}$ (time period $\beta$ ) in Figure 6. A significant level of energy can only be observed after $2800 \mathrm{~s}$ and the corresponding frequency peak related to the maximum AE energy is located around $44 \mathrm{o} \mathrm{kHz}$, time $\mathrm{t}=4000 \mathrm{~s}$. Figure 8 shows also that the energy becomes negligible after $5000 \mathrm{~s}$

The end of the AE activity in the frequency domain $f_{1}$ under investigation could be expected to correspond to the end of the thermal effect. However, as observed above, the outbreak of AE activity at time 2800 s precedes the exothermic effect of the crystallization located around time $3500 \mathrm{~s}$. It seems again reasonable to conclude that $\mathrm{AE}$ allows detecting the onset of nucleation much before the solids generation process becomes significant and, therefore, before its detection using visual or usual basic measurement techniques (e.g. turbidimetry, FBRM, conductimetry, etc.) At time $\mathrm{t}_{2} \cong 4000 \mathrm{~s}$ it was observed that the temperature derivative reaches a maximum that can be assumed to reflect the maximum crystallization rate of $\mathrm{CA}_{\mathrm{a}}$. The same maximum can clearly be related to the sharp peak of absolute energy shown in Figure 8. One can therefore assume that the absolute energy recorded in the frequency domain under investigation is related to the rate of $\mathrm{CA}_{\mathrm{a}}$ production (i.e., crystal growth rather than nucleation).

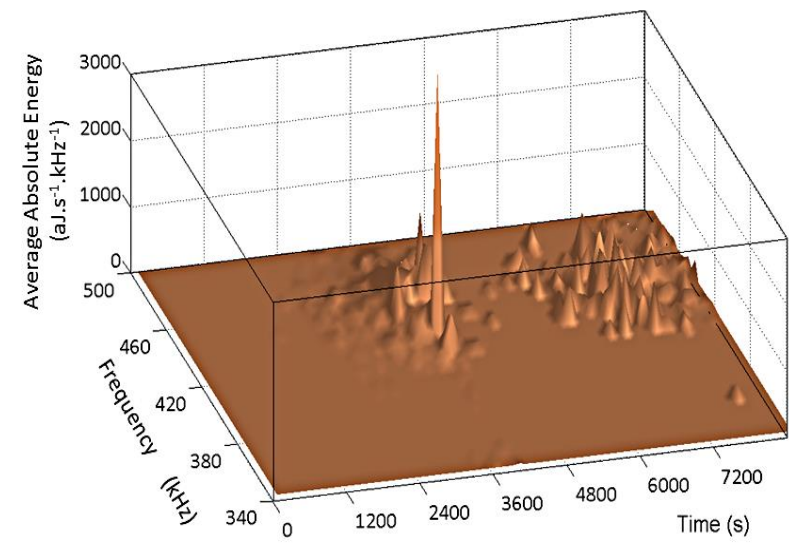

Figure 8. Averaged absolute energy $E_{a b s}$, as a function of the peak frequency and time, computed in the frequency domain spreading from 300 to $500 \mathrm{KHz}$. 
It was also outlined above that many hits recorded in a given frequency region do not necessarily correspond to high $\mathrm{AE}$ energy release. Indeed, many acoustic hits were recorded in the frequency region $f_{1}$ during the time period $\alpha$ but Figure 8 shows that the corresponding average acoustic energy remains quite low (up to $3000 \mathrm{aJ}$ ), when compared to the energy recorded during the crystallization of the monohydrated form of CA.

Now, the crystallization of CA monohydrate occurs as a second process during the same batch operation. It is then important to determine if other characteristic $\mathrm{AE}$ frequencies domains could be used to monitor the crystallization of monohydrate and discriminate between the crystallization of the anhydrous vs. monohydrate forms of CA.

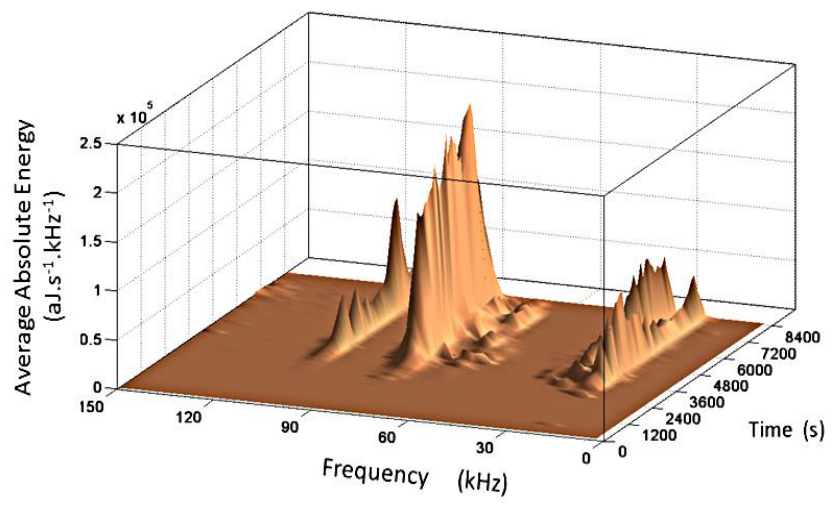

Figure 9. Average absolute energy $E_{a b s}$, as a function of the peak frequency and time, measured in the frequency domain spreading from o to $150 \mathrm{KHz}$.

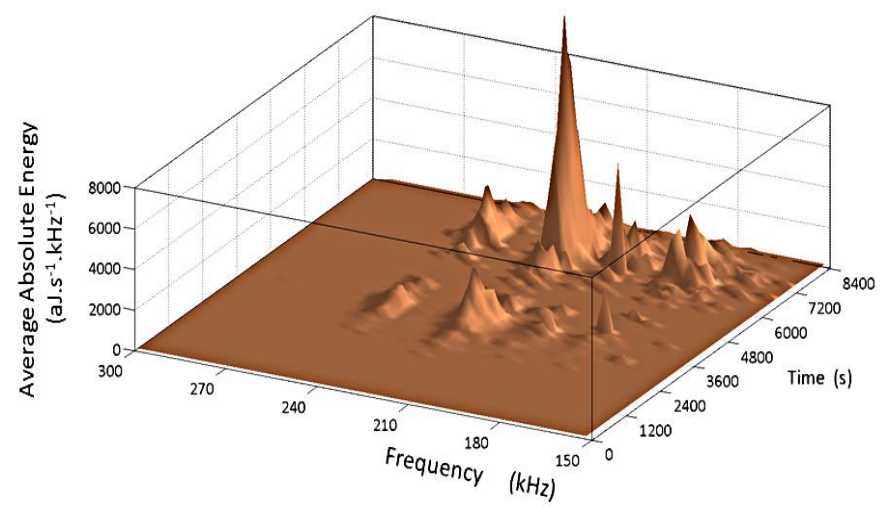

Figure 10. Average absolute energy $E_{a b s}$, as a function of the peak frequency and time, measured in the frequency domain spreading from 150 to $300 \mathrm{KHz}$.

Figure 9 displays a plot of the measured averaged acoustic energy in the frequency interval [o-15o kHz]. This interval corresponds to the frequency bands $f_{6}$ to $f_{9}$ in Figure 6 . During the time period $\delta$, no frequency band can be distinguished that would be specific of the crystallization of a given form of CA. As could already observed, all frequency bands $f_{1}$ to $f_{9}$ start simultaneously at the very first moments of the crystallization of $\mathrm{CA}_{\mathrm{a}}$. The frequency bands $f_{6}$ to $f_{9}$ last throughout the whole crystallization process, except during period $\gamma$ where the acoustic activity is very weak.

The surface plot displayed in Figure 10 shows the energy of the acoustic waves located in the frequency band [150-300 $\mathrm{kHz}$. A very sharp energy peak can be observed during the time period $\delta$. During the same time period (i.e., during the crystallization of $\left(\mathrm{CA}_{\mathrm{m}}\right)$, Figure 7 showed that the acoustic activity recorded in the frequency domain under consideration was much less intense compared to the many events recorded during period $\beta$ (i.e., during the crystallization of $\mathrm{CA}_{\mathrm{a}}$ ). Now, Figure 10 suggests that though it gives rises to less acoustic bursts, the acoustic activity generated by the crystallization of the monohydrate form of CA actually releases more acoustic energy than the crystallization of anhydrous. Such an interesting feature of the acoustic emission could be used to monitor polymorphic transitions occurring during crystallization processes.

To make a deeper analysis of the time/frequen-cy/energy AE features during the whole crystallization process, the time variations of the cumulative absolute energy in the frequency bands studied above were computed. Such calculation results in the 3 plots displayed in Figure 11. These plots allow a closer look at the time variations of the overall average 
acoustic energy and confirms that the generation of monohydrate could be monitored using on-line AE measurements focused on the frequency bands $\mathrm{f}_{2}$ to $\mathrm{f}_{5}$ (i.e., [150-30o] KHz.) Indeed, as one can see in Figure $11 \mathrm{~b}, E_{a b s}$ exhibits two wellseparated peaks corresponding to the generation of the two forms of CA during the solvent-mediated phase transition process. In this frequency area, the second peak is about four times more developed than the peak of anhydrous. This observation suggests that monitoring the appearance of the stable form could be performed through the monitoring of the two peaks observed in Figure $11 \mathrm{~b} \& \mathrm{c}$.
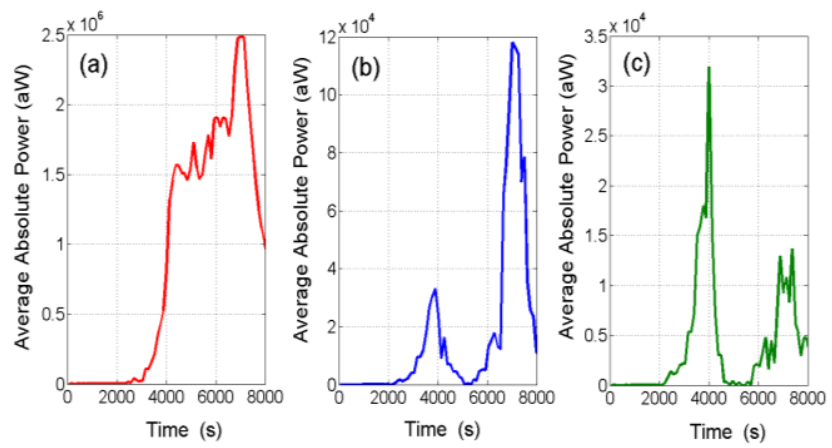

Figure 11. Average integrated absolute energy JEabs vs. time, measured in 3 frequency domains: (a) [o $150 \mathrm{kHz}$; (b) [150 $300 \mathrm{kHz}]$; (c) [300 $500 \mathrm{kHz}$ ]

The sum of absolute acoustic energy on the whole frequency range was also computed so as to evaluate how the overall acoustic energy could be related to the advancement of the crystallization process. Figure 12 shows that the variation of $E_{a b s}$ is monotonously increasing until it reaches a maximum after $7200 \mathrm{~s}$ (Mark (d)). The beginning of the cooling crystallization is clearly identified by mark (a) in Figure 12. Moreover, one can see from the same figure that mark (d) corresponds to a maximal release of heat (i.e., the gap between the setpoint temperature and the actual one is maximal.) One can therefore propose that this maximum corresponds to the maximal rate of monohydrate production. The same observation can be made about the crystallization of $\mathrm{CA}_{\mathrm{a}}$ reflected by a parabolic type profile ending at time $t \approx 4400 \mathrm{~s}($ Mark $b)$. The transition period, including the dissolution of $\mathrm{CA}_{\mathrm{a}}$, can clearly be identified between marks (b) and (c). Even though no solute concentration measurements could be performed during this study, one can suggest that the time variations of $E_{a b s}$ between marks (b) and (c) correspond to the solvent mediated phase transition process during which $\mathrm{CA}_{\mathrm{a}}$ dissolves to allow further $\mathrm{Ca}_{\mathrm{m}}$ nucleation and growth. The principle of such a phase transition process was already explained above and illustrated in Figure 3.

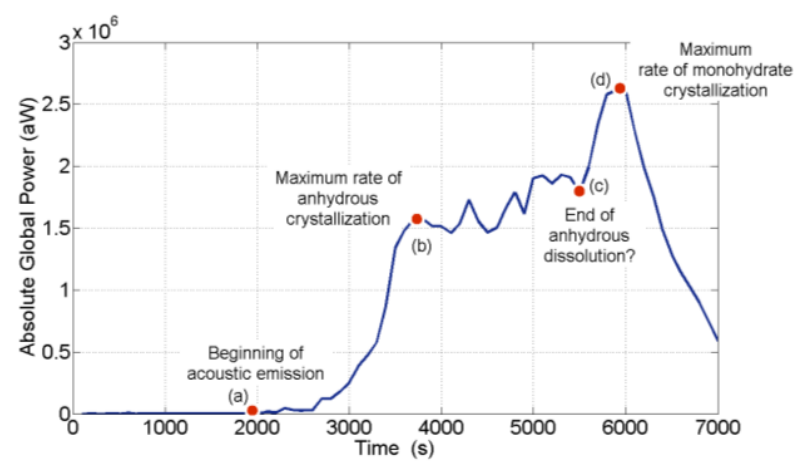

Figure 12. Overall average absolute energy Eabs, as a function of time (i.e., frequency range $(\mathrm{kHz})=\left[\begin{array}{ll}0 & 500\end{array}\right]$ ). The plot shows that the emission starts near $2400 \mathrm{~s}$.

\section{CONCLUSIONS}

In this work, the solvent-mediated phase transition of citric acid during its batch cooling solution crystallization was investigated and monitored thanks to the use of the non-intrusive technique of acoustic emission. First of all, it was clearly shown that meaningful and valuable acoustic signals were emitted during the crystallization process. A first analysis of the time variations of the number of hits recorded throughout the process allowed discriminating between four distinct periods of the batch operation:

- The initial induction period,

- the onset and the development of the crystallization of citric acid anhydrous, 
- a transition period during which both the dissolution of $\mathrm{CA}_{\mathrm{a}}$ and the progressive generation of $\mathrm{Ca}_{\mathrm{m}}$ are likely to occur,

- a period during which the crystallization of citric acid monohydrate is achieved.

Time and frequency analysis of the acoustic signals were shown to provide valuable data allowing a rather easy detection of the nucleation phenomena and of the onset of phase transition between the anhydrous to the monohydrate form of citric acid during the crystallization process. Separating the whole acoustic activity into specific frequency bands allowed a deeper analysis of the phase transition process to be made.

From these observations, it is concluded that:

1. The crystallization of the anhydrous form is accompanied by many acoustic bursts located in the frequency band $\mathrm{f}_{1}$ $(\mathrm{kHz}) \subset\left[\begin{array}{ll}400 & 500\end{array}\right]$, even though the related acoustic absolute energy is not necessarily high.

2. No specific frequency band can be related to the onset of the monohydrate form of CA except "in hollow": crystallization occurring in the absence of activity of the frequency band $\mathrm{f}_{1}$ seems to express specifically the crystallization of $\mathrm{CA}_{\mathrm{m}}$

3. Plotting the cumulated energy recorded in well-chosen relevant frequency bands (see the plots in Figures $11 b$ and c) allows identifying very clearly the development of the two successive crystallization events involved during the crystallization process investigated here.

As far as the monitoring of complex solution crystallization processes is concerned, these preliminary results can be considered as really encouraging and promising for the control of industrial solution crystallization processes, e.g. pharmaceutical crystallization.

\section{REFERENCES}

[1] J. Heinrich, J. Ulrich, Application of Laser-Backscattering Instruments for In Situ Monitoring of Crystallization Processes A Review, Chem. Eng. Technol. 35 (2012) 967-979.

[2] P. Barrett, B. Smith, J. Worlitschek, V. Bracken, B. O'Sullivan, D. O'Grady, A Review of the Use of Process Analytical Technology for the Understanding and Optimization of Production Batch Crystallization Processes, Org. Process Res. Dev. 9 (2005) 348-355.

[3] D.J. McClements, S. Gunasekaran, Ultrasonic characterization of foods and drinks: Principles, methods, and applications. Crit. Rev. Food Sci. Nutr. 37 (1997) 1-46.

[4] W.R. Boyd, J. Varley, The uses of passive measurement of acoustic emissions from chemical engineering processes. Chem. Eng. Sci. 56 (2001) 1749-1767.

[5] E.M. Hansuld, L. Briens, A. Sayani, J.A.B. McCann, An investigation of the relationship between acoustic emissions and particle size. Powder Technol. 219 (2012) 111-117.

[6] T. Jayakumar, C.K. Mukhopadhyay, S. Venugopal, S.L. Mannan, B. Raj, A review of the application of acoustic emission techniques for monitoring forming and grinding processes. J. Mater. Process. Technol. 159 (2005) 48-61.

[7] D. Daniher, L. Briens, A. Tallevi, End-point detection in high-shear granulation using sound and vibration signal analysis. Powder Technol. 181 (2008) 130-136.

[8] E.M. Hansuld, L. Briens, J.A.B. McCann, A. Sayani, Audible acoustics in high-shear wet granulation: Application of frequency filtering. Int. J. Pharm. 378 (2009) 37-44.

[9] M. Whitaker, G.R. Baker, J. Westrup, P.A. Goulding, D.R. Rudd, R.M. Belchamber, M.P. Collins, Application of acoustic emission to the monitoring and end point determination of a high shear granulation process. Int. J. Pharm. 205 (2000) $79-91$.

[10] M. Saeleaw, G. Schleining, Effect of blending cassava starch, rice, waxy rice and wheat flour on physico-chemical properties of flour mixtures and mechanical and sound emission properties of cassava crackers. J. Food Eng. 100 (2010) 12-24.

[11] P. Allan, L.J. Bellamy, A. Nordon, D. Littlejohn, Non-invasive monitoring of the mixing of pharmaceutical powders by broadband acoustic emission. Analyst. 135 (2010) 518-524.

[12] J.T.T. Leskinen, M.-A.H. Okkonen, M.M. Toiviainen, S. Poutiainen, M. Tenhunen, P. Teppola, R. Lappalainen, J. Ketolainen, K. Järvinen, Labscale fluidized bed granulator instrumented with non-invasive process monitoring devices. Chem. Eng. J. 164 (2010) 268-274.

[13] D. Vervloet, J. Nijenhuis, J.R. van Ommen, Monitoring a lab-scale fluidized bed dryer: A comparison between pressure transducers, passive acoustic emissions and vibration measurements. Powder Technol. 197 (2010) 36-48.

[14] S. Poutiainen, S. Matero, T. Hämäläinen, J. Leskinen, J. Ketolainen, K. Järvinen, Predicting granule size distribution of a fluidized bed spray granulation process by regime based PLS modeling of acoustic emission data. Powder Technol. 228 (2012) $149-157$.

[15] M. Halstensen, P. de Bakker, K.H. Esbensen, Acoustic chemometric monitoring of an industrial granulation production process - a PAT feasibility study. Chemom. Intell. Lab. Syst. 84 (2006) 88-97.

[16] E. Rouèche, E. Serris, G. Thomas, L. Périer-Camby, Influence of temperature on the compaction of an organic powder and the mechanical strength of tablets. Powder Technol. 162 (2006) 138-144. 
[17] D.Y.T. Wong, M.J. Waring, P. Wright, M.E. Aulton, Elucidation of the compressive deformation behaviour of $\alpha$-lactose monohydrate and anhydrous $\alpha$-lactose single crystals by mechanical strength and acoustic emission analyses. Int. J. Pharm. 72 (1991) 233-241.

[18] M.J. Waring, M.H. Rubinstein, J.R. Howard, Acoustic emission of pharmaceutical materials: the effect of compression speed, ejection, lubrication and tablet weight. Int. J. Pharm. 40 (1987)15-22.

[19] A.P. Wade, Acoustic emission: is industry listening? Chemom. Intell. Lab. Syst. 8 (1990) 305 - 310.

[20] N. Gherras, E. Serris, G. Fevotte, Monitoring industrial pharmaceutical crystallization processes using acoustic emission in pure and impure media. Int. J. Pharm. 439 (2012) 109-119.

[21] C.U. Grosse, M. Ohtsu, Acoustic Emission Testing, 1st ed. Springer, 2008.

[22] J.K. Burnett, Theory and uses of acoustic emissions, Nova Science Publishers, New York, 2011.

[23] G. Gautschi, Piezoelectric sensorics: force, strain, pressure, acceleration and acoustic emission sensors, materials and amplifiers, Springer, Berlin, New York, 2002.

[24] N. Gherras, E. Serris, G. Fevotte, Monitoring industrial pharmaceutical crystallization processes using acoustic emission in pure and impure media. Int. J. Pharm. 439 (2012) 109-119.

[25] A. Apelblat, E. Manzurola, Solubility of oxalic, malonic, succinic, adipic, maleic, malic, citric, and tartaric acids in water from 278.15 to 338.15 K. J. Chem. Thermodyn. 19 (1987) 317-320.

[26] G. Févotte, C. Alexandre and Sheibat Othman Nida, A population balance model of the solution-mediated phase transition of citric acid. AIChE J. 53 (2007) 2578-2589.

[27] A. Caillet, F. Puel, G. Fevotte, Quantitative in situ monitoring of citric acid phase transition in water using Raman spectroscopy. Chem. Eng. Process. Process Intensif. 47 (2008) 377-382. 